\title{
Structural Determinants of the Capacity
}

\section{of Heparin to Inhibit the Formation of the Human Amplification C3 Convertase}

\author{
Michel D. Kazatchine, Douglas T. Fearon, Dean D. Metcalfe, \\ Robert D. Rosenberg, and K. Frank Austen, Institut National de la Santé \\ et de la Recherche Medicale U 28, Hôpital Broussais, Paris, France; Departments of \\ Medicine, Harvard Medical School, Robert B. Brigham Division of the Affiliated \\ Hospitals Center, Inc., Beth Israel Hospital, and Sidney Farber Cancer Institute, \\ Boston Massachusetts 02115
}

A B S T RACT The ability of heparin glycosaminoglycan to prevent formation of the properdin-stabilized amplification C3 convertase is independent of antithrombin binding activity and requires substitution of the amino sugar and a degree of oxygen $(\mathrm{O})$-sulfation which could be on the uronic acid or the amino sugar. Preparations of heparin glycosaminoglycan isolated by different techniques from different species (rat, human, and porcine) exhibited an equivalent capacity to inhibit generation of the amplification $\mathrm{C} 3$ convertase. Hyaluronic acid, which is devoid of O-sulfation, had no inhibitory activity; chondroitin 4-sulfate of rat and whale origins, chondroitin 6-sulfate of rat and shark origins, and dermatan sulfate from porcine skin are $\mathrm{O}$-sulfated on the galactosamine and had minimal activity. Porcine heparin glycosaminoglycan, isolated on the basis of affinity for antithrombin III, had no greater anticomplementary activity than porcine glycosaminoglycan, which failed to bind antithrombin III and had essentially no anticoagulant activity. Nitrogen (N)-desulfation of porcine heparin reduced anticomplementary activity to the level of the other sulfated mucopolysaccharides, and both $\mathrm{N}$-resulfation and $\mathrm{N}$-acetylation restored the original activities, thereby indicating a requirement for $\mathrm{N}$-substitution, but not $\mathrm{N}$-sulfation. $\mathrm{N}$-resulfation of $\mathrm{N}$-desulfated and $\mathrm{O}$-desulfated heparin did not restore any activity, thus indicating that $\mathrm{O}$-sulfation and $\mathrm{N}$-substitution represent independent, critical structural requirements for the anticomplementary activity of heparin glycosaminoglycan. Inasmuch as $\mathrm{N}$-desulfated-N-acetylated heparin had no anticoagulant

Dr. Fearon is a recipient of Research Career Development Award K04 AI-00245 from the National Institutes of Health.

Received for publication 22 July 1980 and in revised form 25 August 1980. activity, the nature of the $\mathrm{N}$-substitution completely distinguishes the plasma-protein effector pathway that is inhibited.

\section{INTRODUCTION}

Heparin, whether in the fluid phase or particle bound, inhibits activation of the complement system by interfering with formation or regulation of the classical (1-5) or alternative-pathway C3 convertases (6-8), as well as with function of late components (9). Fluid phase heparin prevents generation of the amplification C3 convertase of human complement, $\mathrm{C} 3 \mathrm{~b}, \mathrm{Bb}$, by inhibiting the effective interaction of cell-bound $\mathrm{C} 3 \mathrm{~b}$ with $\mathrm{B}$ and $D(7)$. Inhibition occurs regardless of the presence of properdin $(\mathrm{P}),{ }^{1}$ which stabilizes the amplification convertase. Heparin also prevents formation of the convertase formed in the absence of $\mathrm{D}$ and containing uncleaved B. In a cell-free system with $C 3 b$, heparin impairs the cleavage of $\mathrm{B}$ by $\mathrm{D}$ and does so at concentrations similar to those required for antithrombin III-cofactor activity (7). The inhibitory action of heparin is considered to be on the binding site of C3b for $B$.

To examine the structural requirements for this inhibitory effect, various glycosaminoglycans were compared with heparin and chemically modified de-

\footnotetext{
${ }^{1}$ Abbreviations used in this paper: CPC, cetyl pyridinium chloride; $\mathrm{DGVB}^{++}$, half isotonic $\mathrm{GVB}^{++}$with $2.5 \%$ dextrose; EAC4b,3b, sheep erythrocytes sensitized with specific antibody and bearing the major cleavage fragments of $\mathrm{C} 4$ and $\mathrm{C} 3$; EAC4b,3b, Bb, $P$, cellular intermediate bearing the properdinstabilized amplification C3 convertase (C3,Bb,P); GVB, veronal-buffered saline containing $0.1 \%$ gelatin; $\mathrm{GVB}^{++}$, GVB containing $0.15 \mathrm{mM}$ calcium and $0.5 \mathrm{mM}$ magnesium; GVB-EDTA, GVB containing 0.04 M EDTA; P, properdin; RPMC, rat peritoneal mast cells.
} 
rivatives on the basis of uronic acid content, glucosamine content, or dry weight. Each of the sulfated glycosaminoglycans was inhibitory, and heparin was the most active, suggesting a role for the primary disaccharide structure. For heparin to be active, oxygen (O)-sulfation and substitution of the amino group of glucosamine were absolutely required. There was no relationship between the anticoagulant activity of heparin and the anticomplementary action on the alternative pathway, therefore indicating different structural requirements for the modulation of two different biologic systems.

\section{METHODS}

Materials. Hog intestine heparin (H-3125) with anticoagulant activity of $170 \mathrm{U} / \mathrm{mg}$ (Sigma Chemical Co., St. Louis, Mo.); whale cartilage chondroitin 4-sulfate $(25,000-$ $50,000 \mathrm{~mol} \mathrm{wt})$, shark cartilage chondroitin 6-sulfate $(40,000$ $80,000 \mathrm{~mol} \mathrm{wt}$ ), porcine skin dermatan sulfate, porcine skin hyaluronic acid, chondroitinase ABC from Proteus vulgaris, and chondroitinase AC from Arthrobacter aurescens (Miles Laboratories, Research Products Div., Elkhart, Ind.); Sephadex G-50, Sephadex G-100, and Sepharose 4B (Pharmacia Fine Chemicals, Piscataway, N. J.); Dowex 1-×2 (100-200 mesh, chloride form), Dowex AG 50W- $\times 8$, and DEAE-cellulose (Bio-Rad Laboratories, Richmond, Calif.); azure A and pyridine (Fisher Scientific Co., Fairlawn, N. J.); $\left[{ }^{14} \mathrm{C}\right]$ acetic anhydride (New England Nuclear, Boston, Mass.); sulfurtrioxide trimethylamine complex (Aldrich Chemical Co., Milwaukee, Wis.); Amberlite IRA-400 (Rohm and Haas Co., Philadelphia, Pa.); and fluorescamine (Fluram; Roche Diagnostics Div., Nutley, N. J.), were obtained as indicated.

Preparation of glycosaminoglycans. Purified rat peritoneal mast cells (RPMC) were frozen and thawed six times and extracted with $1 \mathrm{M} \mathrm{NaCl}$; the extract was chromatographed on a Dowex $1-\times 2$ column $(10,11)$. The fractions containing heparin proteoglycan, as assessed by metachromasia (12) and assay of uronic acid content (13), were eluted with $3 \mathrm{M} \mathrm{NaCl}$, dialyzed and lyophilized. This material was treated with chondroitinase $A B C$ as described $(14,15)$ to remove contaminating chondroitin sulfates, and rechromatographed on Dowex 1-×2. The fractions eluted with $3 \mathrm{M} \mathrm{NaCl}$ that contained heparin proteoglycan were pooled, dialyzed against distilled water, and lyophilized. Glycosaminoglycan heparin was prepared by resuspending a portion of the proteoglycan heparin in $0.5 \mathrm{~N} \mathrm{NaOH}$ for $14 \mathrm{~h}$ at room temperature (16). The reaction mixture was neutralized and gel filtered on Sephadex G-50 (15).

To prepare rat cartilage chondroitin sulfate glycosaminoglycans, the xyphoid processes from 100 Sprague-Dawley rats were homogenized by sonication with a Polytron (Kinematic, Lucerne, Switzerland) in $1 \mathrm{M} \mathrm{NaCl}, 0.1 \mathrm{M}$ 6-aminohexanoic acid, $0.005 \mathrm{M}$ benzamidine hydrochloride, and $0.05 \mathrm{M}$ EDTA. The mixture was centrifuged to remove cartilage debris. The supernatant fluid was dialyzed against $1 \mathrm{M} \mathrm{NaCl-0.01} \mathrm{M} \mathrm{Tris,} \mathrm{pH} \mathrm{9.0,} \mathrm{containing} \mathrm{the} \mathrm{protease}$ inhibitors, and the proteoglycans were isolated by Dowex $1-\times 2$ chromatography (17). Rat cartilage glycosaminoglycans were prepared by mild base hydrolysis (16) and isolated by DEAE-cellulose chromatography using logarithmic gradients of $\mathrm{LiCl}(11,17)$. Material containing uronic acid eluted in two peaks that corresponded to the elution positions of whale cartilage chondroitin 4-sulfate and shark cartilage chondroitin 6-sulfate. The ratio of chondroitin 4- to 6-sulfate was 5: 1, based upon chrondroitin AC degradation and analysis by paper chromatography of the separate fractions. Each species of chondroitin sulfate was desalted by Sephadex G-50 gel filtration (17).

Human heparin glycosaminoglycan obtained from lung by Dowex and DEAE-cellulose chromatography exhibited an anticoagulant activity of $137 \mathrm{U} / \mathrm{mg}$ (15).

Fractionation and chemical modification of porcine heparin. Porcine heparin precipitated with cetylpyridinium chloride (CPC) was gel filtered on Sephadex G-100 to obtain pooled fractions of 19,000 and $7,000 \mathrm{~mol}$ wt $(18,19)$. To isolate heparin with a high affinity for antithrombin from each pool, mucopolysaccharide was mixed with antithrombin III at a molar ratio of 1.0:0.08 in 0.01 $\mathrm{M}$ Tris-HCl-0.15 M NaCl, $\mathrm{pH}$ 7.5. High affinity heparin was recovered by gel filtration and was freed of protease inhibitor as described (20). Low affinity heparin was prepared from the material that failed to complex with antithrombin when a molar ratio of $1: 3$ was used to adsorb the mucopolysaccharide. The high affinity and low affinity products were rechromatographed on Sephadex G-100 and shown to be homogeneous in size (20).

A 15,000-mol wt fraction of CPC heparin was used for studies of chemical modifications. $100 \mathrm{mg}$ of CPC heparin in $2 \mathrm{ml}$ of distilled water was filtered through 3-ml Amberlite IRA-400 and $3 \mathrm{ml}$-Dowex $50-\times 8$ columns, and the heparin was neutralized with $0.1 \mathrm{ml}$ of pyridine and lyophilized. 65 $\mathrm{mg}$ of the pyridinium salt of heparin was treated with dimethylsulfoxide containing $5 \%$ methanol for $1.5 \mathrm{~h}$ at $50^{\circ} \mathrm{C}$ to achieve nitrogen (N)-desulfation (21). $40 \mathrm{mg}$ of $\mathrm{N}$-desulfated heparin was then treated with $4 \mathrm{ml}$ of dimethylsulfoxide containing $10 \%$ methanol for $18 \mathrm{~h}$ at $100^{\circ} \mathrm{C}$ to obtain total desulfation (22). N-resulfation of the $\mathrm{N}$-desulfated and the totally desulfated heparins was initiated by adding $5 \mathrm{mg}$ of sulfurtrioxide trimethylamine complex and $5 \mathrm{mg}$ of sodium bicarbonate to $0.3 \mathrm{ml}$ of a $50 \mathrm{mg} / \mathrm{ml}$ solution of each modified heparin at $\mathrm{pH} 9.5$ (23). At the end of the reactions, the products were extensively dialyzed against distilled water and lyophilized. N-desulfated heparin was $\mathrm{N}$-acetylated by reaction with excess $\left[{ }^{14} \mathrm{C}\right]$ acetic anhydride at $\mathrm{pH} 7.5$. The extent of $\mathrm{N}$-acetylation was assessed by incorporation of $\left[{ }^{14} \mathrm{C}\right]$ acetic anhydride and by fluorometric titration of residual amino groups with fluorescamine.

Heparin concentrations were determined by carbazole assay of uronic acid content (13). The levels of chemically modified heparins were quantified by measuring the glucosamine concentration after hydrolysis of samples in $6 \mathrm{~N} \mathrm{HCl}$ for $20 \mathrm{~h}$ as determined in a $121 \mathrm{MB}$ Beckman amino acid analyzer (Beckman Instruments, Spinco Div., Palo Alto, Calif.). Because unsubstituted amine groups were present on the glucosamine residues of the latter components, uronic acid content measured by the carbazole assay is underestimated, as compared with glucosamine measurement. Molecular weights of heparin fractions were determined by gel filtration on Sephadex G-100, calibrated with heparin standards whose molecular weights had been established by analytic ultracentrifugation and light scattering (19). The anticoagulant potency of heparin fractions was determined by their antithrombin III cofactor activity, as compared with a heparin standard of known strength (24). The total sulfate content of heparin was measured (25) in samples filtered through Amberlite IRA-400 and Dowex AG $50 \mathrm{~W}-\times 8$ columns to remove contaminating inorganic ions and hydrolyzed in $2 \mathrm{~N}$ $\mathrm{HCl}$ for $2 \mathrm{~h}$ at $100^{\circ} \mathrm{C}$. The $\mathrm{N}$-sulfate content of heparin was determined by the inorganic sulfate liberated after treating the samples with nitrous acid (26).

Complement components and assays. Components B (27), C3 (28), P (29), and D (30) were purified to homogeneity, as assessed by polyacrylamide gel electrophoresis of their 


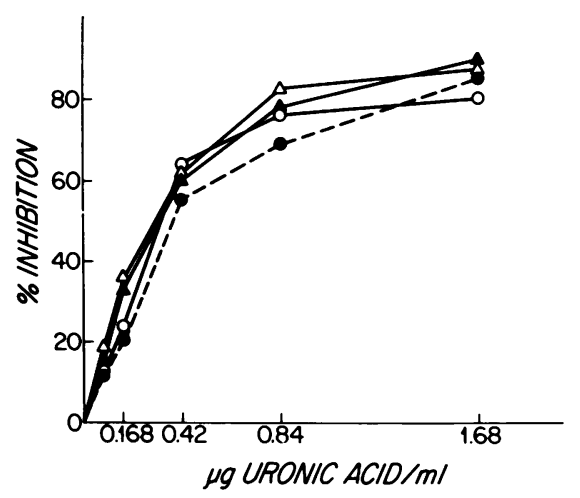

FIGURE 1 Dose-related inhibition of the formation of cellbound P-stabilized $\mathrm{C} 3 \mathrm{~b}, \mathrm{Bb}$ sites by heparin glycosaminoglycan from human lung $(O)$, RPMC $(\triangle)$, and hog intestine $(\Theta)$, and by RPMC proteoglycan $(\boldsymbol{\Lambda})$. Each point represents the mean of duplicate measurements.

reduced forms in the presence of sodium dodecyl sulfate (31). Veronal-buffered saline containing $0.1 \%$ gelatin (GVB), GVB containing $0.15 \mathrm{mM}$ calcium and $0.5 \mathrm{mM}$ magnesium $\left(\mathrm{GVB}^{++}\right)$, half isotonic $\mathrm{GVB}^{++}$with $2.5 \%$ dextrose $\left(\mathrm{DGVB}^{++}\right)$, and GVB containing $0.04 \mathrm{M}$ EDTA (GVB-EDTA) were prepared as described (32).

To examine dose-response effects of mucopolysaccharides on the formation of the P-stabilized amplification C3 convertase, reaction mixtures containing $1 \times 10^{8} \mathrm{EAC} 4 \mathrm{~b}, 3 \mathrm{~b}$ cells/ $\mathrm{ml}, 10 \mathrm{ng} / \mathrm{ml} \mathrm{D}, 65 \mathrm{ng} / \mathrm{ml} \mathrm{P}$, and $0.62-0.83 \mathrm{ng} / \mathrm{ml} \mathrm{B}$ (sufficient to yield approximately one hemolytic site per cell) were prepared in $\mathrm{DGVB}^{++}$at $0^{\circ} \mathrm{C}(30)$. $0.1-\mathrm{ml}$ portions of this

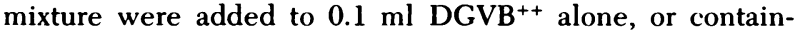
ing incremental concentrations of mucopolysaccharides; the reaction mixtures were incubated for $30 \mathrm{~min}$ at $30^{\circ} \mathrm{C} .0 .3 \mathrm{ml}$ of a 1:20 dilution of rat serum in GVB-EDTA was then added, and incubation was continued for $60 \mathrm{~min}$ at $37^{\circ} \mathrm{C}$. After the addition of $1.6 \mathrm{ml}$ saline, percent lysis was measured, and the average number of hemolytic sites per cell was calculated.

\section{RESULTS}

The inhibitory effects of heparin glycosaminoglycan from different species on the formation of the P-stabilized amplification C3 convertase were compared on the basis of uronic acid content. Heparin glycosaminoglycan from human lung, RPMC, and hog intestine exhibited similar dose-related inhibition of the formation of EAC4b,3b,Bb,P (Fig. 1). Furthermore, on the basis of uronic acid content, there was no difference in inhibitory activity between RPMC-heparin proteoglycan and the glycosaminoglycan side chains cleaved from the proteoglycan (Fig. 1).

Inhibition of P-stabilized amplification C3 convertase formation was also examined using high and low molecular weight species of porcine heparin and their respective fractions with high and low affinity for antithrombin III. The anticoagulant activity ranged from 4 $\mathrm{U} / \mathrm{mg}$ for the fraction with low antithrombin III affinity from the low molecular weight pool to 685 $\mathrm{U} / \mathrm{mg}$ for the fraction with high antithrombin III

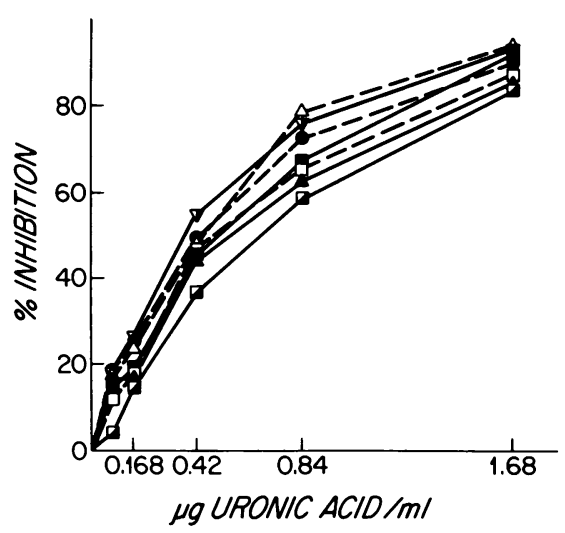

FIGURE 2 Dose-related inhibition of the formation of cellbound P-stabilized C3b,Bb sites by hog heparin glycosaminoglycan (157 U/mg anticoagulant activity) (O), high molecular weight pool (94 U/mg) (G), low molecular weight pool (280

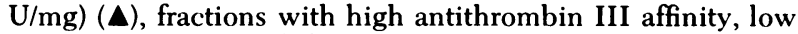
$(350 \mathrm{U} / \mathrm{mg})(\triangle)$ and high molecular weight $(685 \mathrm{U} / \mathrm{mg})$ $(\square)$, respectively, and fractions with low antithrombin III affinity, and low (4 U/mg) $(\nabla)$ and high molecular weight (15 U/mg) ( $\mathbf{D})$, respectively. Each point represents the mean of duplicate measurements.

affinity from the high molecular weight pool. There was, however, essentially no difference in capacity of the various fractions of porcine heparin to inhibit formation of the amplification C3 convertase (Fig. 2).

Because native heparin glycosaminoglycan from different species with different anticoagulant activities had comparable inhibitory activity, the action of glycosaminoglycans with a different disaccharide composition was examined. Chondroitin 4-sulfate from whale or rat cartilage, chondroitin 6-sulfate from shark or rat cartilage and porcine skin dermatan sulfate had minimal inhibitory capacity compared with heparin

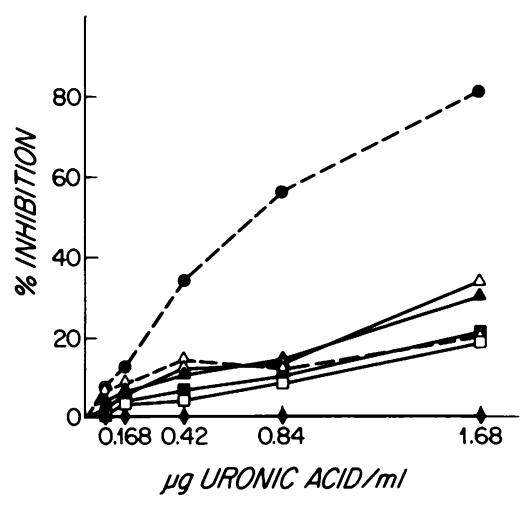

Figure 3 Dose-related inhibition of the formation of cellbound P-stabilized C3b,Bb sites by porcine heparin glycosaminoglycan $(\Theta)$, whale $(\square)$, and rat $(\square)$ cartilage chondroitin 4-sulfate, shark $(\Delta)$, and rat $(\Delta)$ cartilage chondroitin 6-sulfate, porcine dermatan sulfate $(\diamond)$, and hyaluronic acid $(\diamond)$. Each point represents the mean of duplicate measurements. 
TABLE I

Chemical Modifications of $N$ - and O-Sulfate Groups of Heparin*

\begin{tabular}{lll}
\hline & \multicolumn{1}{c}{ N-sulfatest } & \multicolumn{1}{c}{ Total sulfatest } \\
\hline CPC heparin & $0.96(0.91-1.02)$ & $2.69(2.65-2.77)$ \\
N-desulfated & 0 & $1.43(1.42-1.47)$ \\
$\begin{array}{l}\text { N-desulfated } \rightarrow \\
\text { N-resulfated }\end{array}$ & $0.92(0.87-0.95)$ & $2.31(2.23-2.34)$ \\
$\begin{array}{l}\text { N- and O-desulfated } \\
\text { N- and O- }\end{array}$ & 0 & 0 \\
desulfated $\rightarrow$ & & \\
N-resulfated & $0.96(0.96-0.96)$ & $1.03(1.00-1.03)$ \\
\hline
\end{tabular}

* Values shown are the mean of triplicate measurements, with the ranges given in parentheses.

$\ddagger$ Moles $\mathrm{SO}_{4}^{--} /$moles glucosamine.

(Fig. 3). Hyaluronic acid, even at concentrations ten times higher than those noted in Fig. 3, had no effect. When concentrations of glycosaminoglycans sufficient to inhibit $50 \%$ of $\mathrm{C} 3$ convertase formation were compared on a dry weight basis, hog heparin was 10.5, 14.5, and 14 times more potent than chondroitin 4-sulfate from whale cartilage, chondroitin 6-sulfate from shark cartilage, and dermatan sulfate from porcine skin, respectively.

To examine the relative role of $\mathrm{N}$ - and $\mathrm{O}$-sulfate groups in the inhibitory effect of heparin, porcine heparin $(\sim 15,000 \mathrm{~mol} \mathrm{wt})$ was selectively $\mathrm{N}$-desulfated or N- and O-totally desulfated and a portion of each preparation was then $\mathrm{N}$-resulfated (Table I). Another portion of the $\mathrm{N}$-desulfated heparin was $\mathrm{N}$-acetylated, achieving $85 \%$ substitution of the calculated number of free amino groups when assessed by using $\left[{ }^{14} \mathrm{C}\right]-$ acetic anhydride, and $82 \%$ substitution when assessed by fluorometric measurement using fluorescamine of the residual unsubstituted amines. The relative capacity of the modified heparins to inhibit P-stabilized $\mathrm{C} 3 \mathrm{~b}, \mathrm{Bb}$ formation was examined on the basis of their glucosamine content. Through selective $\mathrm{N}$-desulfation, inhibitory activity of the starting heparin almost ceased, whereas $\mathrm{N}$-resulfation restored the original activity (Fig. 4A). $\mathrm{N}$-acetylation of $\mathrm{N}$-desulfated heparin was also sufficient to restore the original inhibitory activity (Fig. 4B). The marked loss of activity with $\mathrm{N}$ - and $\mathrm{O}$ desulfation was not reversed by $\mathrm{N}$-resulfation alone (Fig. 4A).

\section{DISCUSSION}

The capacity of heparin glycosaminoglycan to inhibit the generation of the amplification C3 convertase of complement (7) has been further characterized by identifying certain of the primary structural requirements for those effects. Heparin glycosaminoglycan obtained from three different species, pig intestine, human lung, and purified RPMC exhibited comparable inhibitory activity when normalized for uronic acid content (Fig. 1). Fractions of porcine glycosaminoglycan (19,000 and 7,000 mol wt, respectively) had the same inhibitory activity on the generation of the alternative pathway amplification C 3 convertase, but differed by a factor of 3 in their anti-coagulant activity

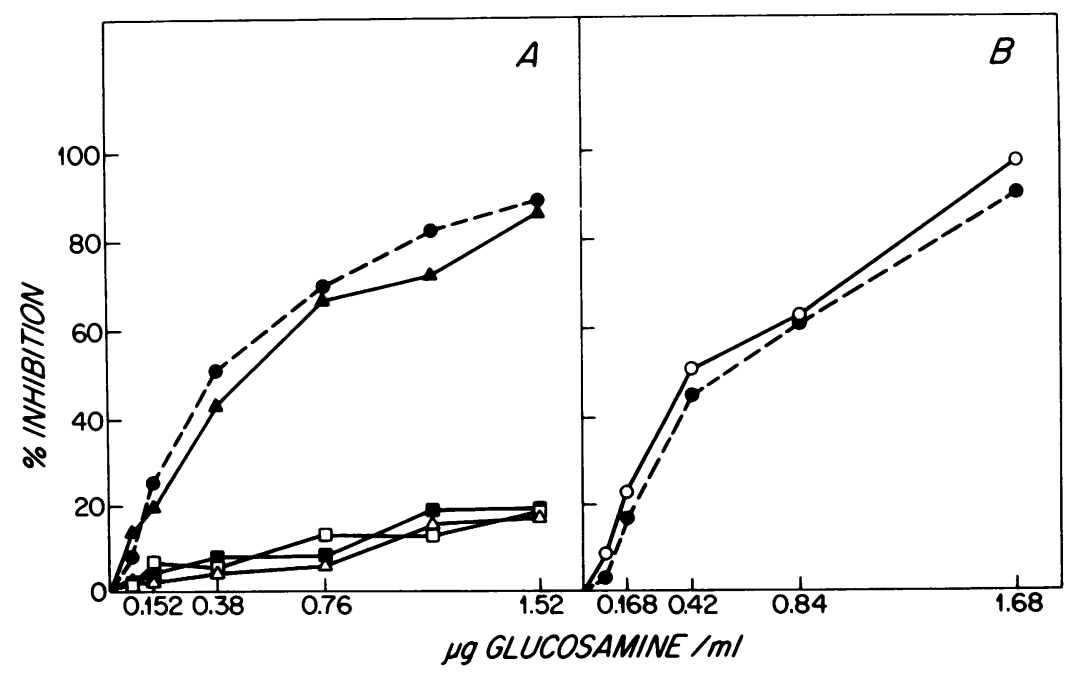

FIgURE 4 Dose-related inhibition of the formation of cell-bound P-stabilized C3b,Bb sites (panel A) by porcine heparin glycosaminoglycan $(205 \mathrm{U} / \mathrm{mg})(0), \mathrm{N}$-desulfated heparin $(0 \mathrm{U} / \mathrm{mg})$ $(\Delta), \mathrm{N}$-desulfated, N-resulfated heparin $(61.9 \mathrm{U} / \mathrm{mg})(\Delta), \mathrm{N}$ - and O-desulfated heparin $(0 \mathrm{U} / \mathrm{mg})$ $(\square)$, and $\mathrm{N}$ - and O-desulfated, N-resulfated heparin $(0 \mathrm{U} / \mathrm{mg})(\square)$, and (panel B) CPC heparin (O) and N-desulfated N-acetylated heparin (0 U/mg) (O). 
(Fig. 2 and Table II). Subfractions of each pool, isolated by their high affinity for antithrombin III, had the same relative inhibitory activity as subfractions from each pool with little or no affinity for antithrombin III. Thus, the fractions from the $19,000-\mathrm{mol}$ wt porcine glycosaminoglycan pool, with high affinity for antithrombin III and an anticoagulant activity of $685 \mathrm{U} / \mathrm{mg}$, had the same inhibitory activity based on uronic acid, glucosamine content, or dry weight, as a fraction from the 7,000-mol wt pool with no apparent affinity for antithrombin III and an anticoagulant activity of 4 $\mathrm{U} / \mathrm{mg}$ (Table II). The tetrasaccharide sequence in the fractions with high affinity for antithrombin III, Liduronic acid $\rightarrow \mathrm{N}$-acetylated D-glucosamine-6sulfate $\rightarrow$ D-glucuronic acid $\rightarrow \mathrm{N}$-sulfated D-glucosamine-6-sulfate (33), is apparently not critical to the activity of the heparin glycosaminoglycan that inhibits generation of the amplification C3 convertase of complement.

Either N-desulfation, or a combination of $\mathrm{N}$ - and O-desulfation, removed the inhibitory activity of porcine heparin. In as much as either $\mathrm{N}$-resulfation, or $\mathrm{N}$-acetylation, restored the inhibitory activity of $\mathrm{N}$ desulfated heparin to normal, the substitution of the free amino group is essential to the anticomplementary activity, possibly to prevent neutralization of negatively charged $\mathrm{O}$-sulfates. In contrast, acetylation of $\mathrm{N}$-desulfated heparin did not restore any anticoagulant activity, indicating the importance of sulfation of the amino sugar for this activity. N-resulfation did not regenerate any activity from the $\mathrm{N}$ - and $\mathrm{O}$-desulfated heparin, indicating that $\mathrm{O}$-sulfation, as well as $\mathrm{N}$-substitution is essential for full anticomplementary activity of porcine heparin glycosaminoglycan (Fig. 4, Table II).

Because $\mathrm{N}$-desulfated, $\mathrm{N}$-acetylated porcine heparin was as active as native or $\mathrm{N}$-resulfated heparin, and much more active than chondroitin 4- or 6-sulfate or dermatan sulfate, it is possible that 6-sulfated glucosamine rather than 4-sulfated galactosamine is an important component. Another possibility is that $\mathrm{O}$-sulfation of the uronic acid rather than $\mathrm{O}$-sulfation of the particular stereoisomeric form of the amino sugar is important for anticomplementary activity.

Hyaluronic acid (GlcUA $\rightarrow$ GlcNAc disaccharide), which is devoid of O-sulfation, had no inhibitory activity, whereas the mucopolysaccharides sulfated only in the amino sugars, such as chondroitin 4-sulfate (GlcUA $\rightarrow$ 4- $\mathrm{SO}_{3}$-GalNAc disaccharide), chondroitin 6-sulfate (GlcUA $\rightarrow$ 6- $\mathrm{SO}_{3}$-GalNAc disaccharide) and dermatan sulfate (IdUA $\rightarrow$ 4- $\mathrm{SO}_{3}$-GalNAc disaccharide) had low activity, suggesting that $\mathrm{O}$-sulfation of the uronic acid may be needed for substantial anticomplementary activity. The higher degree of O-sulfation of heparin, which has $1.4-1.7$ sulfates per disaccharide, compared with either whale chondroitin
TABLE II

Anticoagulant and Anticomplementary Activities of Heparin Species of Different Molecular Weight or Affinity for Antithrombin III and of Chemically Modified Heparins

\begin{tabular}{lcc}
\hline & $\begin{array}{c}\text { Anticoagulant } \\
\text { activity }\end{array}$ & $\begin{array}{c}\text { Inhibitory activity on } \\
\text { convertase formation } \\
\text { relative to heparin* }\end{array}$ \\
\hline USP U/mg & \\
Porcine heparin & & \\
glycosaminoglycan & 205.4 & 1.00 \\
HMW pool & 94.0 & 0.92 \\
LMW pool & 280.0 & 0.92 \\
High affinity HMW & 685.0 & 0.88 \\
Low affinity HMW & 15.0 & 0.84 \\
High affinity LMW & 350.0 & 0.96 \\
Low affinity LMW & 4.0 & 1.10 \\
N-desulfated & 0.0 & 0.13 \\
N-desulfated $\rightarrow$ N- & & \\
resulfated & 61.9 & 0.98 \\
N- and O-desulfated & 0.0 & 0.13 \\
N- and O-desulfated $\rightarrow$ N- & & \\
$\quad$ resulfated & 0.0 & 0.15 \\
N-desulfated $\rightarrow$ N- & & 1.10 \\
$\quad$ acetylated & 0.0 & \\
\hline
\end{tabular}

* Relative inhibitory activity is based on concentration required to achieve $50 \%$ inhibition of convertase formation. The data for the chemically modified heparins are derived from Fig. 4 and the other data are derived from the mean of three experiments including that depicted in Fig. 2.

† HMW, high molecular weight; LMW, low molecular weight.

4-sulfate or shark chondroitin 6-sulfate, which have an average of 1.0 sulfate per disaccharide, may also contribute to its greater inhibitory activity. However, as heparin was approximately eight times more active than the mucopolysaccharides containing $\mathrm{O}$-sulfated $\mathrm{N}$-acetyl galactosamine (Fig. 3), it is again suggested that $\mathrm{O}$-sulfation of the uronic acid is important for anticomplementary activity.

Inasmuch as the metabolism of native heparin proteoglycan, which is the form in which the molecule would be secreted from the mast cells (34), is not established, it is noteworthy that the activity of the rat heparin proteoglycan was the same as that of the rat glycosaminoglycan in terms of uronic acid content. The ability of the anaphylatoxic cleavage fragments of the third and fifth complement proteins to initiate granule secretion from mast cells (35) suggests that the inhibitory action of heparin on the amplification $\mathrm{C} 3$ convertase of complement has a negative feedback role.

\section{ACKNOWLEDGMENTS}

We acknowledge the excellent technical assistance of Ms. Barbara Reaves.

This work was supported by grants AI-07722, AM-05577, RR-05669, and HL-19131 from the National Institutes of 
Health, a grant from the New England Peabody Home for Crippled Children, and a grant from the Delegation Generale a la Recherche Scientifique et Technique, France.

\section{REFERENCES}

1. Ecker, E. E., and P. Gross. 1929. Anticomplementary power of heparin. J. Infect. Dis. 44: 250-253.

2. Raepple, E., H. -U. Hill, and M. Loos. 1976. Mode of interaction of different polyanions with the first $(\mathrm{Cl}$, $\mathrm{C} \overline{1})$, the second $(\mathrm{C} 2)$ and the fourth (C4) component of complement. I. Effect on fluid phase $C \overline{1}$ and on $C \overline{1}$ bound to EA or to EAC4. Immunochemistry. 13: 251-2.55.

3. Loos, M., J. E. Volanakis, and R. M. Stroud. 1976. Mode of interaction of different polyanions with the first $(\mathrm{Cl}, \mathrm{CI})$, the second (C2) and the fourth (C4) component of complement. III. Inhibition of $\mathrm{C} 4$ and $\mathrm{C} 2$ binding site(s) on Cls̄ by polvanions. Immunochemistry. 13: 789-791.

4. Loos, M., J. E. Volanakis, and R. M. Stroud. 1976. Mode of interaction of different polyanions with the first $(\mathrm{C} 1, \mathrm{C} \overline{\mathrm{l}})$, the second $(\mathrm{C} 2)$ and the fourth $(\mathrm{C} 4)$ component of complement. II. Effect of polyanions on the binding of C2 to EAC4b. Immunochemistry. 13: 257261.

5. Rent, R., R. Myhrman, B. A., Fiedel, and H. Gewurz. 1976. Potentiation of Cl-esterase inhibitory activity by heparin. Clin. Exp. Immunol. 23: 264-271.

6. Brai, M., and A. G. Osler. 1972. Studies of the C3 shunt activation in cobra venom-induced lysis of unsensitized erythrocytes. Proc. Soc. Exp. Biol. Med. 140: 1116-1121.

7. Weiler, J. M., R. W. Yurt, D. T. Fearon, and K. F. Austen. 1978. Modulation of the formation of the amplification convertase of complement, $\mathrm{C} 3 \mathrm{~b}, \mathrm{Bb}$, by native and commercial heparin. J. Exp. Med. 147: 409-421.

8. Kazatchkine, M. D., D. T. Fearon, J. E. Silbert, and K. F. Austen. 1979. Surface-associated heparin inhibits zymosaninduced activation of the human alternative complement pathway by augmenting the regulatory action of the control proteins on particle-bound C3b. J. Exp. Med. 150: 1202-1215.

9. Baker, P. J., T. F. Lint, B. C. McLeod, C. L. Behrends, and $\mathrm{H}$. Gewurz. 1975. Studies on the inhibition of C $\overline{56}-$ induced lysis (reactive lysis). VI. Modulation of C $\overline{56}$ induced lysis by polyanions and polycations. J. Immunol. 114: 554-558.

10. Slorach, S. A. 1971. Histamine and heparin release from isolated rat mast cells exposed to compound 48/80. Acta Physiol. Scand. 82: 91-97.

11. Yurt, R. W., R. W. Leid, K. F. Austen, and J. E. Silbert. 1977. Native heparin from rat peritoneal mast cells. J. Biol. Chem. 252: 518-521.

12. Jaques, L. B., F. C. Monkhouse, and M. Stewart. 1949. A method for the determination of heparin in blood. $J$. Physiol. (Paris). 109: 41-48.

13. Bitter, T., and H. Muir. 1962. A modified uronic acid carbazole reaction. Anal. Biochem. 4: 330-334.

14. Yamagata, T., H. Siato, O. Habuchi, and S. Suzuki. 1968. Purification and properties of bacterial chondroitinases and chondrosulfatases. J. Biol. Chem. 243: 1523-1535.

15. Metcalfe, D. D., R. A. Lewis, J. E. Silbert, R. D. Rosenberg, S. I. Wasserman, and K. F. Austen. 1979. Isolation and characterization of heparin from human lung. $J$. Clin. Invest. 64: 1537-1543.

16. Robinson, H. C., A. A. Horner, M. Hook, S. Ogren, and U. Lindahl. 1978. A proteoglycan form of heparin and its degradation to single-chain molecules. J. Biol. Chem. 253: $6687-6693$.

17. Metcalfe, D. D., S. I. Wasserman, and K. F. Austen. 1980.
Isolation and characterization of sulfated mucopolysaccharides from rat basophil leukemia (RBL-1) cells. Biochem. J. 185: 367-382.

18. Rosenberg, R. D., G. Armand, and L. H. Lam. 1978. Structure-function relationships of heparin species. Proc. Natl. Acad. Sci. U. S. A. 75: 3065-3069.

19. Salzman, E. W., R. D. Rosenberg, M. H. Smith, J. N. Lindon, and L. Favreau. 1980. Effect of heparin and heparin fractions on platelet aggregation. J. Clin. Invest. 65: $64-73$.

20. Jordan, R., D. Beeler, and R. D. Rosenberg. 1979. Fractionation of low molecular weight heparin species and their interaction with antithrombin. J. Biol. Chem. 254: 2902-2913.

21. Inoue, Y., and K. Nagasawa. 1976. Selective N-desulfation of heparin with dimethyl sulfoxide containing water or methanol. Carbohydr. Res. 46: 87-95.

22. Nagasawa, K., Y. Inoue, and T. Kamata. 1977. Solvolytic desulfation of glycosaminoglycuronan sulfates with dimethyl sulfoxide containing water or methanol. Carbohydr. Res. 58: 47-55.

23. Lloyd, A. G., G. Embery, and L. J. Fowler. 1971. Studies on heparin degradation. I. Preparation of $\left({ }^{35} \mathrm{~S}\right)$ sulfamate derivatives for studies on heparin degrading enzymes of mammalian origin. Biochem. Pharmacol. 20: 637-648.

24. Lam, L. H., J. E. Silbert, and R. D. Rosenberg. 1976. The separation of active and inactive forms of heparin. Biochem. Biophys. Res. Commun. 69: 570-577.

25. Dodgson, K. S. 1961. Determination of inorganic sulphate in studies on the enzymatic and non-enzymatic hydrolysis of carbohydrate and other sulphate esters. Biochem. J. 78: 312-319.

26. Inoue, Y., and K. Nagasawa. 1976. A new method for the determination of $\mathrm{N}$-sulfate in heparin and its analogs. Anal. Biochem. 71: 46-52.

27. Hunsicker, L. G., S. Ruddy, and K. F. Austen. 1973. Alternate complement pathway: factors involved in cobra venom factor (CoVF) activation of the third component of complement (C3). J. Immunol. 110: 128-138.

28. Tack, B. B., and J. W. Prahl. 1976. Third component of human complement: purification from plasma and physicochemical characterization. Biochemistry. 15: 4513-4521.

29. Fearon, D. T., and K. F. Austen. 1977. Activation of the alternative complement pathway due to resistance of zymosan-bound amplification convertase to endogenous regulatory mechanisms. Proc. Natl. Acad. Sci. U. S. A. 74: 1683-1687.

30. Fearon, D. T., and K. F. Austen. 1975. Properdin: binding to $\mathrm{C} 3 \mathrm{~b}$ and stabilization of the C3b-dependent C3 convertase. J. Exp. Med. 142: 856-863.

31. Weber, K., and M. Osborn. 1969. The reliability of molecular weight determinations by dodecyl sulfate polyacrylamide gel electrophoresis. J. Biol. Chem. 244: 4406-4412.

32. Nelson, R. A., J. Jensen, I. Gigli, and N. Tamura. 1966. Methods for the separation, purification and measurement of the nine components of hemolytic complement in guinea pig serum. Immunochemistry. 3: 111-135.

33. Rosenberg, R. D., and L. Lam. 1979. Correlation between structure and function of heparin. Proc. Natl. Acad. Sci. U. S. A. 76: 1218-1222.

34. Yurt, R. W., R. W. Leid, Jr., J. Spragg, and K. F. Austen. 1977. Immunologic release of heparin from purified rat peritoneal mast cells. J. Immunol. 118: 1201-1207.

35. Dias da Silva, W., and I. H. Lepow. 1967. Complement as a mediator of inflammation. II. Biological properties of anaphylatoxin prepared with purified components of human complement. J. Exp. Med. 125: 921-946. 